\title{
DIVISIBILITY PARAMETERS AND THE DEGREE OF KUMMER EXTENSIONS OF NUMBER FIELDS
}

\author{
Antonella Perucca - Pietro Sgobba - Sebastiano Tronto \\ Department of Mathematics, Faculty of Science, Technology and Medicine, \\ University of Luxembourg, LUXEMBOURG
}

\begin{abstract}
Let $K$ be a number field, and let $\ell$ be a prime number. Fix some elements $\alpha_{1}, \ldots, \alpha_{r}$ of $K^{\times}$which generate a subgroup of $K^{\times}$of rank $r$. Let $n_{1}, \ldots, n_{r}, m$ be positive integers with $m \geqslant n_{i}$ for every $i$. We show that there exist computable parametric formulas (involving only a finite case distinction) to express the degree of the Kummer extension $K\left(\zeta_{\ell^{m}}, \sqrt[\ell_{1}^{n_{1}}]{\alpha_{1}}, \ldots, \sqrt[\ell]{n_{r}}{ }_{\alpha_{r}}\right)$ over $K\left(\zeta_{\ell} m\right)$ for all $n_{1}, \ldots, n_{r}, m$. This is achieved with a new method with respect to a previous work, namely we determine explicit formulas for the divisibility parameters which come into play.
\end{abstract}

\section{Introduction}

Let $K$ be a number field, and let $\ell$ be a prime number. Fix some elements $\alpha_{1}, \ldots, \alpha_{r}$ of $K^{\times}$which generate a (without loss of generality) torsion-free subgroup of $K^{\times}$of rank $r$. Since this is a natural question in Kummer theory, we are interested in computing the degree of the Kummer extension

$$
K\left(\zeta_{\ell^{m}}, \sqrt[\ell^{n_{1}}]{\alpha_{1}}, \ldots, \sqrt[\ell^{n_{r}}]{\alpha_{r}}\right) / K\left(\zeta_{\ell^{m}}\right)
$$

for all positive integers $n_{1}, \ldots, n_{r}, m$ with $m \geqslant n_{i}$ for every $i$, where $\zeta_{\ell^{m}}$ denotes a primitive $\ell^{m}$ th root of unity. In a recent work [4] we have proven that it is

(C) 2021 BOKU-University of Natural Resources and Life Sciences and Mathematical Institute, Slovak Academy of Sciences.

2010 Mathematics Subject Classification: Primary: 11Y40; Secondary: 11R20, 11R21. Keywords: number field, Kummer theory, Kummer extension, degree.

(c) $(1) \Theta \Theta$ Licensed under the Creative Commons BY-NC-ND 4.0 International Public License. 


\section{ANTONELLA PERUCCA—PIETRO SGOBBA-SEBASTIANO TRONTO}

possible to compute parametric formulas (involving only a finite case distinction) to express the degree of the extensions (11) for all $n_{1}, \ldots, n_{r}, m$. We now achieve this result in a more direct way. Namely, setting $n=\max \left(n_{1}, \ldots, n_{r}\right)$ we can write the above extension as

$$
K\left(\zeta_{\ell^{m}}, \sqrt[\ell^{n}]{\alpha_{1}^{\ell^{n-n_{1}}}}, \ldots, \sqrt[\ell^{n}]{\alpha_{r}^{\ell^{n-n_{r}}}}\right) / K\left(\zeta_{\ell^{m}}\right)
$$

By the results in [1, Section 3.3], the degree of this extension is known to depend only on certain finitely many computable divisibility parameters for the group

$$
\left\langle\alpha_{1}^{\ell^{n-n_{1}}}, \ldots, \alpha_{r}^{\ell^{n-n_{r}}}\right\rangle
$$

So we want to determine these divisibility parameters as $n_{1}, \ldots, n_{r}$ vary.

More precisely, given a finitely generated and torsion-free subgroup $G$ of $K^{\times}$of positive rank $r$, we consider two types of parameters expressing the $\ell$-divisibility of $G$ in $K$ : the $d$-parameters are non-negative integers $d_{1}, \ldots, d_{r}$, while the $h$-parameters are non-negative integers $h_{1}, \ldots, h_{r}$ that can be at most the $\ell$-adic valuation of $\# \mu_{K}$, where $\mu_{K}$ is the group of roots of unity in $K$. We formally define these notions, as well as the notion of strongly $\ell$-independent elements, in Section 2 .

The main result we achieve is the following (see Theorem 4.4).

TheORem 1.1. Let $K$ be a number field, and let $\ell$ be a prime number. Fix some elements $\alpha_{1}, \ldots, \alpha_{r}$ of $K^{\times}$which generate a torsion-free subgroup of $K^{\times}$ of rank $r$. There is a finite procedure to determine formulas for the $\ell$-divisibility parameters of the group

$$
\left\langle\alpha_{1}^{\ell^{x_{1}}}, \ldots, \alpha_{r}^{\ell^{x_{r}}}\right\rangle
$$

for all non-negative integers $x_{1}, \ldots, x_{r}$. There is a finite partition of the set of $r$-tuples $x_{1}, \ldots, x_{r}$ such that, if we restrict to any subset of the partition, the $d$-parameters $d_{i}$ are such that $\delta_{i}:=d_{i}-x_{i}$ are fixed integers, where $i \in$ $\{1, \ldots, r\}$, and the corresponding $h$-parameters are fixed integers $\varepsilon_{i}$.

We also prove various assertions for the $\ell$-divisibility parameters of the groups (44) and for the degrees of the Kummer extensions (11). In particular, we prove the following result, where $v_{\ell}$ denotes the $\ell$-adic valuation over $\mathbb{Q}$.

TheOREM 1.2. Let $K$ be a number field, and let $\ell$ be a prime number such that $\ell \neq 2$ or $\zeta_{4} \in K$. Let $\omega$ be the largest integer such that $K\left(\zeta_{\ell}\right)=K\left(\zeta_{\ell \omega}\right)$. Fix some elements $\alpha_{1}, \ldots, \alpha_{r}$ of $K^{\times}$which generate a torsion-free subgroup of $K^{\times}$of rank $r$. Then for all $n_{1}, \ldots, n_{r}, m$ with $m \geq n:=\max _{i}\left(n_{i}\right)$ and $m \geq \omega$ 
we have

$$
\begin{aligned}
v_{\ell}\left[K\left(\zeta_{\ell^{m}}, \sqrt[n_{1}]{\alpha_{1}}, \ldots, \sqrt[\ell^{n_{r}}]{\alpha_{r}}\right): K\left(\zeta_{\ell^{m}}\right)\right] & \\
=\max \left\{0, n-m+\varepsilon_{i}-\max \left(n_{i}-\delta_{i}, 0\right):\right. & 1 \leq i \leq r\} \\
& +\sum_{i=1}^{r} \max \left(n_{i}-\delta_{i}, 0\right),
\end{aligned}
$$

where $\delta_{1}, \ldots, \delta_{r}$ and $\varepsilon_{1}, \ldots, \varepsilon_{r}$ are as in Theorem 1.1 (setting $\left.x_{i}:=n-n_{i}\right)$.

In Section 5 we explain how we can compute the degrees of the Kummer extensions (11) in the case $\ell=2$ and $\zeta_{4} \notin K$. More precisely, we describe how the 2-divisibility parameters of a group of the form (44) in $K^{\times}$change from $K$ to $K\left(\zeta_{4}\right)$. Finally, in Section 6. we provide explicit formulas for the $\ell$-divisibility parameters of a torsion-free subgroup of $K^{\times}$of rank at most 3 .

As an aside remark, notice that it is possible to consider the degree of

$$
K\left(\zeta_{\ell^{m}}, \sqrt[n^{n_{1}}]{\alpha_{1}}, \ldots, \sqrt[\ell^{n_{r}}]{\alpha_{r}}\right)
$$

also over $K$, because cyclotomic degrees are easy to compute. For the same reason we could suppose without loss of generality that the elements $\alpha_{1}, \ldots, \alpha_{r}$ generate a torsion-free subgroup of $K^{\times}$.

\section{Divisibility parameters}

Let $K$ be a number field, and let $\ell$ be a prime number. Let $\mu_{K}$ be the group of roots of unity in $K$ and define $z:=v_{\ell}\left(\# \mu_{K}\right)$, where $v_{\ell}$ is the $\ell$-adic valuation over $\mathbb{Q}$. If $\zeta$ is a root of unity, then we denote by $\operatorname{ord}(\zeta)$ its order.

An element $a \in K^{\times}$is called strongly $\ell$-indivisible if there is no root of unity $\zeta$ in $K$ (whose order we may suppose to be a power of $\ell$ ) such that $\zeta a$ is an $\ell$ th power in $K^{\times}$. We call $a_{1}, \ldots, a_{r} \in K^{\times}$strongly $\ell$-independent if $a_{1}^{x_{1}} \cdots a_{r}^{x_{r}}$ is strongly $\ell$-indivisible whenever $x_{1}, \ldots, x_{r}$ are integers not all divisible by $\ell$.

Let $G$ be a finitely generated and torsion-free subgroup of $K^{\times}$of positive rank $r$. If $g_{1}, \ldots, g_{r}$ is a basis of $G$ as a free $\mathbb{Z}$-module, then we can write

$$
g_{i}=\xi_{i} \cdot b_{i}^{\ell^{d_{i}}}
$$

for some strongly $\ell$-indivisible elements $b_{i} \in K^{\times}$, for some integers $d_{i} \geq 0$, and for some $\xi_{i} \in \mu_{K}$ of order $\ell^{h_{i}}$. We call $d_{i}$ and $h_{i}$ the $\ell$-divisibility parameters of $g_{i}$, and we call $b_{i}$ the strongly $\ell$-indivisible part of $g_{i}$. In general, $b_{i}$ and $h_{i}$ may depend on the chosen decomposition: if $h_{i} \leq z-d_{i}$, then there is another decomposition for which $h_{i}=0$; if $h_{i}>z-d_{i}$, then $h_{i}$ is unique. 


\section{ANTONELLA PERUCCA—PIETRO SGOBBA-SEBASTIANO TRONTO}

We call $g_{1}, \ldots, g_{r}$ an $\ell$-good basis of $G$ if $b_{1}, \ldots, b_{r}$ are strongly $\ell$-independent or, equivalently, if the sum $\sum_{i} d_{i}$ is maximal among the possible bases of $G$, see [1. Section 3.1]. In this case, we call $d_{i}$ and $h_{i}$ the $d$-parameters and $h$-parameters for the $\ell$-divisibility of $G$ in $K$. The $d$-parameters of $G$ are unique up to reordering, while in general the $h$-parameters are not unique (one may require additional conditions as to make them unique, see Remark 3). Recall from [1, Theorem 14] that an $\ell$-good basis of $G$ always exists, and from [1, Section 6.1 ] that the $\ell$-divisibility parameters are computable.

LEMMA 2.1. Let $A_{1}, \ldots, A_{r}$ be elements of $K^{\times}$which are strongly $\ell$-independent. Then for all non-zero integers $X_{1}, \ldots, X_{r}$ and for every $\ell^{z}$ th root of unity $\zeta$, the element $\zeta \prod_{i=1}^{r} A_{i}^{X_{i}}$ has d-parameter $\min _{i}\left(v_{\ell}\left(X_{i}\right)\right)$ and h-parameter $v_{\ell}(\operatorname{ord}(\zeta))$.

Proof. Set $d:=\min _{i}\left(v_{\ell}\left(X_{i}\right)\right)$. The element

$$
A:=\prod_{i=1}^{r} A_{i}^{X_{i} / \ell^{d}}
$$

is the product of powers of strongly $\ell$-independent elements whose exponents are not all divisible by $\ell$ and hence it is strongly $\ell$-indivisible. So we can write

$$
\zeta \prod_{i=1}^{r} A_{i}^{X_{i}}=\zeta A^{\ell^{d}}
$$

and from the latter decomposition we can easily read off the $\ell$-divisibility parameters.

Lemma 2.2 ([1, Corollary 16]). The d-parameters $d_{1}, \ldots, d_{r}$ for $G$ are such that for every sufficiently large integer $n$ we have

$$
\frac{G}{G \cap\left(K^{\times}\right)^{\ell^{n}}} \cong \bigoplus_{i=1}^{r} \frac{\mathbb{Z}}{\ell^{n-d_{i}} \mathbb{Z}} .
$$

REMARK 1. If $H$ is a subgroup of $G$ of finite index, then the $d$-parameters for $H$ are greater than or equal to the $d$-parameters for $G$. More precisely, if

$$
d_{1}, \ldots, d_{r} \quad \text { and } \quad d_{1}^{\prime}, \ldots, d_{r}^{\prime}
$$

are the $d$-parameters (ordered non-decreasingly) for $G$ and $H$, then we have

$$
d_{i} \leq d_{i}^{\prime} \quad \text { for all } \quad i \in\{1, \ldots, r\} .
$$


Indeed, if $n$ is sufficiently large, then we have

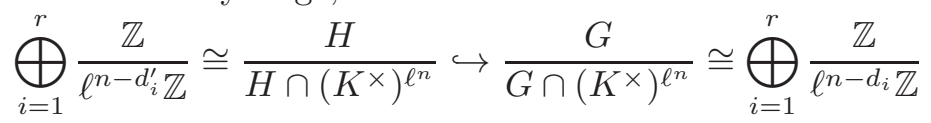

considering that the group homomorphism

$$
H \hookrightarrow G / G \cap\left(K^{\times}\right)^{\ell^{n}} \quad \text { has kernel } H \cap\left(K^{\times}\right)^{\ell^{n}} .
$$

If $m$ is an integer coprime to $\ell$, then clearly the groups $G$ and $G^{m}$ have the same $\ell$-divisibility parameters. This observation and Remark 1 give

REMARK 2. If $H$ is a subgroup of $G$ of index coprime to $\ell$, then the $d$-parameters for $H$ are the same as those for $G$.

REMARK 3. Let $d_{i}$ with $i \in\{1, \ldots, r\}$ be the $d$-parameters of $G$, ordered nondecreasingly. By [1, Appendix] the corresponding $h$-parameters $h_{i}$, for an appropriate choice of the $\ell$-good basis of $G$, satisfy the following conditions (which mean that the $h$-parameters are taken zero whenever possible) and in this case the multiset of the pairs $\left(d_{i}, h_{i}\right)$ is unique:

(1) For every $1 \leqslant i \leqslant r$ we have $h_{i}=0$ or $h_{i}>z-d_{i}$.

(2) If $1 \leqslant i<j \leqslant r$ and $h_{i}, h_{j}>0$ hold, then we have $h_{i}>h_{j}$ and $d_{i}+h_{i}<d_{j}+h_{j}$.

(3) If $1 \leqslant i<j \leqslant r$ and $d_{i}=d_{j}$ hold, then $h_{j}=0$.

To obtain an $\ell$-good basis as above there is provided an algorithm in [1, Proposition 31].

\section{An intrinsic description for the $h$-parameters}

Let $K$ be a number field, let $\ell$ be a prime number, and let $G$ be a torsion-free and finitely generated subgroup of $K^{\times}$of positive rank $r$.

REMARK 4. If $H$ is a subgroup of $G$ of finite index, then it is not true, in general, that the $h$-parameters (as in Remark 3) do not increase from $G$ to $H$. For example, let $a_{1}, a_{2}$ be strongly $\ell$-independent elements of $K^{\times}$, and let $n \geq 1$, $z \geq 2$ : considering

$$
G:=\left\langle a_{1}^{\ell^{n}}, \zeta_{\ell^{z}} a_{2}^{\ell^{z+n}}\right\rangle \quad H:=\left\langle\zeta_{\ell^{z}}\left(a_{1} a_{2}^{\ell^{z}}\right)^{\ell^{n}}, \zeta_{\ell^{z-1}} a_{2}^{\ell^{z+n+1}}\right\rangle
$$

the $h$-parameters of $G$ are $(0, z)$, whereas the $h$-parameters of $H$ are $(z, z-1)$. 


\section{ANTONELLA PERUCCA—PIETRO SGOBBA-SEBASTIANO TRONTO}

However, in general we have the following property.

Lemмa 3.1. Let $H$ be a subgroup of $G$ of finite index, and let $h_{1}, \ldots, h_{r}$ (respectively, $h_{1}^{\prime}, \ldots, h_{r}^{\prime}$ ) be the $h$-parameters as in Remark 3 for $G$ (respectively, $H)$. Then for every $i \in\{1, \ldots, r\}$ we have $h_{i}=0$ or $h_{i}^{\prime} \leq h_{i}$.

P r o o f. Let $g_{1}, \ldots, g_{r}$ and $\gamma_{1}, \ldots, \gamma_{r}$ be $\ell$-good bases for $G$ and $H$, respectively, such that their $d$-parameters $d_{i}$ 's and $d_{i}^{\prime}$ 's are ordered non-decreasingly and their $h$-parameters $h_{i}$ 's and $h_{i}^{\prime}$ 's satisfy the conditions of Remark 3. There are integers $e_{i j}$, roots of unity $\xi_{j} \in \mu_{K}$ of order $\ell^{h_{j}}$, and strongly $\ell$-independent elements $b_{j} \in K^{\times}$such that for every $i \in\{1, \ldots, r\}$ we can write

$$
\gamma_{i}=\prod_{j=1}^{r} g_{j}^{e_{i j}}=\prod_{j=1}^{r} \xi_{j}^{e_{i j}} \cdot b_{j}^{e_{i j} \ell^{d_{j}}} .
$$

Suppose that $h_{i}, h_{i}^{\prime}$ are both strictly positive for some index $i$. Then $h_{i}^{\prime}>z-d_{i}^{\prime}$ and it does not depend on the decomposition, so we have $h_{i}^{\prime}=v_{\ell}\left(\right.$ ord $\left.\prod_{j} \xi_{j}^{e_{i j}}\right)$. To prove that this number is at most $h_{i}$, we show that $h_{j}-v_{\ell}\left(e_{i j}\right)<h_{i}$ for all $j \neq i$ with $h_{j} \neq 0$ and $e_{i j} \neq 0$. By Condition (2) of Remark 3 we have: if $j>i$, then $h_{j}<h_{i}$; If $j<i$, then $h_{j}+d_{j}<h_{i}+d_{i}$, and we conclude because $v_{\ell}\left(e_{i j}\right)+d_{j} \geq d_{i}^{\prime} \geq d_{i}$ by Lemma 2.1 and Remark 1

We can prove a stronger property if we consider a subgroup of $G$ of index coprime to $\ell$.

TheOREM 3.2. If $H$ is a subgroup of $G$ of index coprime to $\ell$, then the $h$ -parameters (as in Remark [3) are the same for $H$ and for $G$.

P r o o f. Let $m$ be the index of $H$ in $G$. By Remark 2 the $d$-parameters $d_{i}$ (in nondecreasing order) are the same for $G, H$, and $G^{m}$. Call $h_{i}$ the corresponding $h$ -parameters (as in Remark 3) for $G$, which are the same for $G^{m}$, and call $h_{i}^{\prime}$ the $h$-parameters for $H$. By Lemma 3.1 (applied once to $G, H$ and once to $H, G^{m}$ ) we know that if $h_{i}, h_{i}^{\prime}$ are both non-zero, then $h_{i}=h_{i}^{\prime}$. It suffices to show that $h_{i}^{\prime}=0$ implies $h_{i}=0$ because the other implication can be proven analogously (replacing $G, H$ by $H, G^{m}$ ). So suppose that $h_{i}^{\prime}=0$ and $h_{i}>0$ for some index $i$.

We keep the notation of the proof of Lemma 3.1. We claim that there is some index $s$ such that $d_{s}=d_{i}$ (hence $h_{s}^{\prime}=0$ ) and $v_{\ell}\left(e_{s i}\right)=0$. Since ord $\xi_{i}^{e_{s i}}=\ell^{h_{i}}$, by reasoning as in Lemma 3.1 we deduce that

$$
v_{\ell}\left(\operatorname{ord} \prod_{j=1}^{r} \xi_{j}^{e_{s j}}\right)=h_{i} .
$$


This is impossible because $h_{i}>z-d_{i}=z-d_{s}$ while the left-hand side is a possible $h$-parameter for $\gamma_{s}$ so it is at most $z-d_{s}$.

To prove the claim, consider the matrix $\left(e_{i j}\right)$, which has determinant coprime to $\ell$, and decompose it in blocks by grouping together indices for which the $d$ -parameters are the same. By Lemma 2.1 we have either $e_{i j}=0$ or $v_{\ell}\left(e_{i j}\right) \geq d_{i}$, and in particular the blocks below the main diagonal are zero modulo $\ell$. Then each square block on the main diagonal has determinant coprime to $\ell$ and the claim follows.

\section{The algorithm for the divisibility parameters}

Let $K$ be a number field, and let $\ell$ be a prime number. Fix some $\zeta \in \mu_{K}$ of order $\ell^{z}$. Let

$$
G:=\left\langle g_{1}, \ldots, g_{r}\right\rangle
$$

be a torsion-free subgroup of $K^{\times}$of positive rank $r$. Choosing an $\ell$-good basis for $G$ and considering the $\ell$-indivisible parts of the elements of this basis, we find strongly $\ell$-independent elements $b_{1}, \ldots, b_{r}$ of $K^{\times}$such that for all $i \in\{1, \ldots, r\}$ we have

$$
g_{i}:=\zeta^{f_{i}} \cdot \prod_{j=1}^{r} b_{j}^{e_{i j}},
$$

where $e_{i j}$ and $f_{i}$ are integers, the matrix $\left(e_{i j}\right)$ has rank $r$, and $1 \leq f_{i} \leq \ell^{z}$.

Lemma 4.1. Suppose that for every $i, j \in\{1, \ldots, r\}$ we have: $e_{i j}=0$ for all $j<i ; e_{i i} \neq 0 ; v_{\ell}\left(e_{i i}\right)=\min _{j \geq i}\left(v_{\ell}\left(e_{i j}\right)\right.$ ) (where we restrict to the indices such that $\left.e_{i j} \neq 0\right)$. Then the $g_{i}$ 's are an $\ell$-good basis of $G$.

P r o of. By Lemma 2.1 the strongly $\ell$-indivisible part of $g_{i}$ is

$$
B_{i}:=\prod_{j \geq i} b_{j}^{e_{i j} \ell^{-v_{\ell}\left(e_{i i}\right)}} .
$$

To prove that the $B_{i}$ 's are strongly $\ell$-independent, suppose that $\xi \prod_{i} B_{i}^{z_{i}}$ is an $\ell$ th power in $K^{\times}$for some integers $z_{i}$ and for some $\xi \in \mu_{K}$, and write

$$
\prod_{i} B_{i}^{z_{i}}=\prod_{j} b_{j}^{n_{j}}, \quad \text { where } \quad n_{j}:=\sum_{i \leq j} e_{i j} \ell^{-v_{\ell}\left(e_{i i}\right)} z_{i} .
$$

Since the $b_{j}$ 's are strongly $\ell$-independent, we have $\ell \mid n_{j}$ for every $j$. In particular, since $\ell$ divides $n_{1}$ but not $e_{11} \ell^{-v_{\ell}\left(e_{11}\right)}$, we have $\ell \mid z_{1}$, and then by iteration we deduce that $\ell \mid z_{i}$ for all $i$. 


\section{ANTONELLA PERUCCA—PIETRO SGOBBA-SEBASTIANO TRONTO}

LEMMA 4.2. The smallest $d$-parameter for $G$ is the minimum of $v_{\ell}\left(e_{i j}\right)$ (where we restrict to the indices such that $e_{i j} \neq 0$ ).

Proof. Let $m:=v_{\ell}\left(e_{i_{0} j_{0}}\right)$ be this minimum. Then all elements of $G$ are $\ell^{m}$ th powers in $K^{\times}$up to a root of unity, and hence every $d$-parameter is at least $m$. By Lemma 2.1 the $d$-parameter of $g_{i_{0}}$ equals $m$, so not all $d$-parameters are greater than $m$.

For every $r$-tuple $X=\left(x_{1}, \ldots, x_{r}\right)$ of non-negative integers define the subgroup

$$
H_{X}:=\left\langle g_{1}^{\ell^{x_{1}}}, \ldots, g_{r}^{\ell^{x_{r}}}\right\rangle
$$

of $G$, which is again torsion-free and of rank $r$.

If $x_{i}+v_{\ell}\left(f_{i}\right) \geq z$ for every $i$, then by Lemma 2.1 the $h$-parameters of the elements $g_{i}^{\ell^{x_{i}}}$ 's are zero and hence we can take the $h$-parameters of $H_{X}$ to be all zero.

REMARK 5. If the $g_{i}$ 's are an $\ell$-good basis of $G$, then the $g_{i}^{\ell^{x_{i}}}$ 's are an $\ell$-good basis for $H_{X}$. So by Lemma 2.1 the group $H_{X}$ has $d$-parameters $d_{i}=\min _{j}\left(v_{\ell}\left(e_{i j}\right)+x_{i}\right)$ (where we restrict to the indices such that $e_{i j} \neq 0$ ) and corresponding $h$-parameters $h_{i}=\max \left(0, z-x_{i}-v_{\ell}\left(f_{i}\right)\right)$.

From now on we order tuples of integers of the same length in lexicographic order.

Definition 4.3. Given the $\left(r+r^{2}+r\right)$-tuple $\left(x_{i}, e_{i j}, f_{i}\right)$, we consider a permutation $\sigma_{I}$ on the the indices $i$ and a permutation $\sigma_{J}$ of the indices $j$ and we define

$$
\sigma\left(x_{i}, e_{i j}, f_{i}\right)=\left(x_{\sigma_{I}(i)}, e_{\sigma_{I}(i) \sigma_{J}(j)}, f_{\sigma_{I}(i)}\right) .
$$

For every $k \in\{1, \ldots, r\}$ we define the following $k^{2}$-tuple (ordered according to $(a, b))$

$$
\sigma_{k}\left(e_{i j}\right):=\left(e_{\sigma_{I}^{-1}(a) \sigma_{J}^{-1}(b)}\right) \quad a, b \in\{1, \ldots, k\} .
$$

We similarly define the $\left(k^{2}+k\right)$-tuple $\sigma_{k}\left(e_{i j}, f_{i}\right)$.

ThEOREM 4.4. There is a finite procedure to determine $\ell$-divisibility parameters of $H_{X}$ for all $X$. The parameters only depend on the $\left(r+r^{2}+r\right)$-tuple $\left(x_{i}, e_{i j}, f_{i}\right)$ (and the d-parameters do not depend on $f_{i}$ ).

There is a permutation $\sigma$ as in Definition 4.3 (which depends only on the $\left(r+r^{2}\right)$-tuple $\left.\left(x_{i}, e_{i j}\right)\right)$, and for every $k \in\{1, \ldots, r\}$ there are two functions $\delta_{k}$ and $\epsilon_{k}$ (which depend only on $k$, and which only involve sums, differences, products, and the $\ell$-adic valuation) such that

$d_{k}:=x_{\sigma_{I}^{-1}(k)}+\delta_{k}\left(\sigma_{k}\left(e_{i j}\right)\right) \quad$ and $\quad h_{k}:=\max \left(0, z-x_{\sigma_{I}^{-1}(k)}-\epsilon_{k}\left(\sigma_{k}\left(e_{i j}, f_{i}\right)\right)\right)$ 
are $\ell$-divisibility parameters of $H_{X}$, with $d_{1}, \ldots, d_{r}$ in non-decreasing order. The above formulas for all $X$ involve a finite case distinction, which means (considering the $e_{i j}$ as fixed) partitioning the set of $r$-tuples $X$ according to $\sigma$.

We can choose the functions $\delta_{k}$ in such a way that we can determine $\sigma$ as follows. We determine $\left(\sigma_{I}^{-1}(k), \sigma_{J}^{-1}(k)\right)$ for all $k$. For $k=1$, we choose the smallest pair of indices (for which $\delta_{1}$ is well-defined) minimizing $d_{1}$. For $k>1$ we consider the formula for $d_{k}$ (where we have already fixed $\left(\sigma_{I}^{-1}(i), \sigma_{J}^{-1}(i)\right.$ ) for all $i<k$ ) and select indices from the remaining ones: we choose the smallest pair of indices (for which $\delta_{k}$ is well-defined) minimizing $d_{k}$.

The proof of the theorem outlines what we can choose as functions $\delta_{k}$ and $\epsilon_{k}$. The fact that $\delta_{k}$ is not well-defined for all integer values of the variables is only due to the fact that the function involves the $\ell$-adic valuation and we do not admit the expression $v_{\ell}(0)$. Expressions for $\delta_{k}$ and $\epsilon_{k}$ for $k$ up to 3 can be found in Section 6

REMARK 6. The algorithm in the proof of the theorem transforms the augmented matrix $\left(e_{i j} \ell^{x_{i}} \mid f_{i} \ell^{x_{i}}\right)$ into a matrix $\left(E_{i j} \mid F_{i}\right)$, where the square submatrix $\left(E_{i j}\right)$ is upper-triangular. The $d$-parameters of $H_{X}$ (in non-decreasing order) are $v_{\ell}\left(E_{i i}\right)$, and the corresponding $h$-parameters are $v_{\ell}\left(\operatorname{ord} \zeta^{F_{i}}\right)$. The finite case distinction yielding $\sigma$ arises from the fact that we permute rows and columns of the matrix to work with entries having least $\ell$-adic valuation (among a subset of the non-zero entries).

P r o of. Consider the $r \times(r+1)$ integer matrix $\left(e_{i j} \ell^{x_{i}} \mid f_{i} \ell^{x_{i}}\right)$, and notice that this matrix without the last column has rank $r$.

STEP 1. Suppose that $e_{11} \ell^{x_{1}}$ is non-zero and has minimal $\ell$-adic valuation among the non-zero entries of the first $r$ columns. Then

$$
\begin{aligned}
& d_{1}:=v_{\ell}\left(e_{11} \ell^{x_{1}}\right)=x_{1}+v_{\ell}\left(e_{11}\right) \\
& h_{1}:=v_{\ell}\left(\operatorname{ord}\left(\zeta^{f_{1} \ell^{x_{1}}}\right)\right)=\max \left(0, z-x_{1}-v_{\ell}\left(f_{1}\right)\right)
\end{aligned}
$$

are the smallest $d$-parameter of $H_{X}$ (by Lemma 4.2) and a corresponding $h$ -parameter (by the meaning of the last column).

If the above assumption is not satisfied, let $\left(i_{1}, j_{1}\right)$ be the smallest pair of indices with $i_{1}, j_{1} \in\{1, \ldots, r\}$ such that $e_{i_{1} j_{1}} \ell^{x_{i_{1}}}$ is non-zero and has minimal $\ell$-adic valuation. Then we swap the rows $1, i_{1}$ and the columns $1, j_{1}$ and rename the indices according to these swaps. 


\section{ANTONELLA PERUCCA—PIETRO SGOBBA-SEBASTIANO TRONTO}

Set $y:=\ell^{-v_{\ell}\left(e_{11}\right)}$. We multiply all rows $i \geq 2$ by $e_{11} y$, which corresponds to replacing $H_{X}$ with a subgroup $H_{X}^{\prime}$ of index coprime to $\ell$. Next, for each $i \geq 2$ we subtract a suitable integer multiple (namely, $y e_{i 1} \ell^{x_{i}-x_{1}}$ ) of row 1 from row $i$, in such a way that the entry $(i, 1)$ of the matrix becomes zero. This corresponds to a base change for the group $H_{X}^{\prime}$. We obtain the matrix

$$
\left(\begin{array}{cccc|c}
e_{11} \ell^{x_{1}} & e_{12} \ell^{x_{1}} & \cdots & e_{1 r} \ell^{x_{1}} & f_{1} \ell^{x_{1}} \\
0 & y \Delta_{22} \ell^{x_{2}} & \cdots & y \Delta_{2 r} \ell^{x_{2}} & y\left(e_{11} f_{2}-e_{21} f_{1}\right) \ell^{x_{2}} \\
\vdots & \vdots & & \vdots & \vdots \\
0 & y \Delta_{r 2} \ell^{x_{r}} & \cdots & y \Delta_{r r} \ell^{x_{r}} & y\left(e_{11} f_{r}-e_{r 1} f_{1}\right) \ell^{x_{r}}
\end{array}\right)
$$

where

$$
\Delta_{i j}=e_{11} e_{i j}-e_{i 1} e_{1 j}
$$

For $i \in\{2, \ldots, r\}$ and $j \in\{2, \ldots, r+1\}$, the $(i, j)$ entry only depends on

$$
e_{11}, e_{i 1}, e_{1 j}, e_{i j}, f_{1}, f_{i}, x_{i}
$$

(and we can remove $f_{i}$ from the above list if $j \neq r+1$ ).

SteP 2. Let $X_{2}=\left(x_{2}, \ldots, x_{r}\right)$. The submatrix obtained by deleting the first row and the first column corresponds to a torsion-free subgroup

$$
H_{X_{2}} \text { of }\left\langle b_{2}, \ldots, b_{r}, \mu_{\ell^{z}}\right\rangle \text { of rank } r-1
$$

whose generating elements are $\ell^{x_{i}}$ th powers (for $i \geq 2$ ) of elements of $K^{\times}$that do not depend on the $x_{i}$ 's. We proceed as above. We suppose that the entry $(1,1)$ of the submatrix is non-zero and has minimal $\ell$-adic valuation (among the nonzero entries of its first $r-1$ columns), else we permute rows and columns of the original matrix (not the first row, and neither the first nor the last column) to achieve this condition, and rename the indices according to these swaps. Then the smallest $d$-parameter for $H_{X_{2}}$ and a corresponding $h$-parameter are

$$
\begin{aligned}
d_{2} & :=v_{\ell}\left(y\left(e_{11} e_{22}-e_{21} e_{12}\right) \ell^{x_{2}}\right) \\
& =x_{2}-v_{\ell}\left(e_{11}\right)+v_{\ell}\left(e_{11} e_{22}-e_{21} e_{12}\right) \\
h_{2} & :=v_{\ell}\left(\operatorname{ord}\left(\zeta^{y\left(e_{11} f_{2}-e_{21} f_{1}\right) \ell^{x_{2}}}\right)\right) \\
& =\max \left(0, z-x_{2}+v_{\ell}\left(e_{11}\right)-v_{\ell}\left(e_{11} f_{2}-e_{21} f_{1}\right)\right) .
\end{aligned}
$$

We modify the matrix as above to have zeroes in the second column below the diagonal. 
STEP $k$ (for $3 \leq k \leq r$ ). Following this procedure, by iteration we find expressions for the functions $d_{k}, h_{k}, \delta_{k}, \epsilon_{k}$ of the desired form (up to permuting the indices, in their expressions we only find indices $i, j \leq k$ ).

At Step $k$ we swap only rows and columns starting from the $k$-th ones, and we never affect the $(r+1)$-st column: the composition of these transpositions for the rows and for the columns is what we call $\sigma_{I}$ and $\sigma_{J}$ respectively. The assertion about determining $\sigma$ follows from the fact that at the $k$ th step the quantity that we want to be minimal is precisely the value that we take for $d_{k}$ (by definition of $\left.d_{k}\right)$.

The first $r$ columns of the final matrix form an upper triangular matrix. The integers $d_{k}$ are in non-decreasing order (recall that $d_{1}$ was the smallest $d$ -parameter) because the entries of the submatrix at Step $k$ for $k \geq 2$ are integer combinations of entries of the submatrix at Step $k-1$ (which are either 0 or have $\ell$-adic valuation at least $d_{k-1}$ ).

To conclude, we show that the $d_{k}$ 's and the $h_{k}$ 's are the divisibility parameters for $H_{X}$. The elements described by the rows of the final matrix are a basis $\gamma_{1}, \ldots, \gamma_{r}$ for a subgroup of $H_{X}$ of index coprime to $\ell$ (which has the same divisibility parameters as $H_{X}$ by Theorem 3.2). This is an $\ell$-good basis by Lemma 4.1. and $d_{k}, h_{k}$ are the divisibility parameters of $\gamma_{k}$ by Lemma 2.1.

REMARK 7. In the proof of Theorem 4.4 we transform a matrix, and at all steps the entries are integers of the following form: a function of the $e_{i j}$ 's times $\ell^{x_{k}}$ for some $k$ (the index $k$ is the same for each row and different rows have different indices). We consider the non-zero entries of a submatrix and compare their $\ell$-adic valuations. This amounts to selecting two non-zero entries at a time and considering inequalities of the type

$$
x_{k_{1}}-x_{k_{2}} \leq f\left(e_{i j}\right)
$$

for some function $f$ which depends only on the two entries that we are comparing, at which step we are in the algorithm, and on $\sigma$ (if $r$ is fixed, then there are only finitely many possibilities for the function $f$ ).

Pro of of The or em 1.1. This is a consequence of Theorem 4.4

Proof of Theorem 1.2. Given $\ell$-divisibility parameters for the group

$$
\left\langle\alpha_{1}^{\ell^{n-n_{1}}}, \ldots, \alpha_{r}^{\ell^{n-n_{r}}}\right\rangle
$$

we can apply [1, Theorem 18] to compute the degree of (2). To compute the parameters we can apply Theorem 1.1 (setting $x_{i}:=n-n_{i}$ ). 


\section{ANTONELLA PERUCCA—PIETRO SGOBBA-SEBASTIANO TRONTO}

\section{The 2-divisibility parameters over $K\left(\zeta_{4}\right)$}

Theorem 1.2 excludes the case $\ell=2$ and $\zeta_{4} \notin K$. If $\zeta_{4} \notin K$, then in order to compute the degree of

$$
K\left(\zeta_{2^{m}}, \sqrt[2^{n_{1}}]{\alpha_{1}}, \ldots \sqrt[2^{n_{r}}]{\alpha_{r}}\right) / K\left(\zeta_{2^{m}}\right)
$$

with $m \geq 2$ we may replace $K$ with $K\left(\zeta_{4}\right)$ and apply Theorem 1.2 to compute the 2-divisibility parameters of the group

$$
\left\langle\alpha_{1}^{2^{n-n_{1}}}, \ldots, \alpha_{r}^{2^{n-n_{r}}}\right\rangle \text { over } K\left(\zeta_{4}\right) .
$$

For $m=1$ we can make use of [1, Lemma 19], which also requires the computation of degrees over $K\left(\zeta_{4}\right)$. In this section, we explain how the 2-divisibility parameters of a group change when extending $K$ to $K\left(\zeta_{4}\right)$.

By [3] the 2-divisibility parameters of a finitely generated and torsion-free subgroup of $K^{\times}$change from $K$ to $K\left(\zeta_{4}\right)$ only if

$$
K \cap \mathbb{Q}\left(\zeta_{2^{\infty}}\right)=\mathbb{Q}\left(\zeta_{2^{s}}+\zeta_{2^{s}}^{-1}\right)
$$

holds for some $s \geq 2$. So we consider a number field $K$ satisfying (8) and we set

$$
f:=\zeta_{2^{s}}+\zeta_{2^{s}}^{-1}+2=\zeta_{2^{s}}^{-1}\left(1+\zeta_{2^{s}}\right)^{2} .
$$

We also fix a torsion-free subgroup

$$
G:=\left\langle g_{1}, \ldots, g_{r}\right\rangle
$$

of $K^{\times}$of rank $r$ and set

$$
H_{X}:=\left\langle g_{1}^{2^{x_{1}}}, \ldots, g_{r}^{2^{x_{r}}}\right\rangle,
$$

where $X=\left(x_{1}, \ldots, x_{r}\right)$ is an $r$-tuple of nonnegative integers.

Lemma 5.1. The 2-divisibility parameters of $H_{X}$ change from $K$ to $K\left(\zeta_{4}\right)$ if and only if the same holds for $G$. Equivalently, $H_{X}$ contains an element of the form $\pm\left(f a^{2}\right)^{2^{d}}$ for some $a \in K^{\times}$and for some integer $d \geq 0$ if and only if $G$ contains an element of such form.

Proof. By [3, Theorem 1] the $d$-parameters for $G$ (respectively, $H_{X}$ ) change from $K$ to $K\left(\zeta_{4}\right)$ if and only if $G$ (respectively, $H_{X}$ ) contains an element of the prescribed form. Since $H_{X} \subseteq G$, it is sufficient to prove that if $G$ contains such an element, then so does $H_{X}$. Suppose that

$$
\pm\left(f a^{2}\right)^{2^{d}}=\prod_{i=1}^{r} g_{i}^{e_{i}}
$$


for some integers $e_{i}$. If w.l.o.g. we have $x:=\max _{i}\left(x_{i}\right) \geq 1$, then $H_{X}$ contains

$$
\left(f a^{2}\right)^{2^{d+x}}=\prod_{i=1}^{r}\left(g_{i}^{2^{x_{i}}}\right)^{e_{i} 2^{x-x_{i}}} .
$$

For the remaining of the section suppose that $G$ contains an element as in Lemma 5.1, so that the 2-divisibility parameters for $H_{X}$ change from $K$ to $K\left(\zeta_{4}\right)$. Let $d_{i}, h_{i}$ be the $d$-parameters and $h$-parameters for $H_{X}$ over $K$, see Theorem 4.4. By [3, Theorems 1 and 2] we have:

REMARK 8. There is $j \in\{1, \ldots, r\}$ such that the $d$-parameters for $H_{X}$ over $K\left(\zeta_{4}\right)$ are the $d_{i}$ 's for $i \neq j$ and $d_{j}+1$, while the $h$-parameters for $H_{X}$ over $K\left(\zeta_{4}\right)$ are the $h_{i}$ 's for $i \neq j$ and

$$
\begin{array}{cl}
h_{j} & \text { if } d_{j} \geq s, \\
0 & \text { if } d_{j}=s-1 \quad \text { and } \quad h_{j}=1, \\
1 & \text { if } d_{j}=s-1 \text { and } h_{j}=0, \\
s-d_{j} & \text { if } d_{j} \leq s-2 .
\end{array}
$$

ThEOREM 5.2. There is a finite procedure, not requiring computations over $K\left(\zeta_{4}\right)$, to determine formulas (involving only a finite case distinction) for the 2-divisibility parameters for $H_{X}$ over $K\left(\zeta_{4}\right)$ for all $X$.

P r o of. We can express the elements $g_{i}$ 's in terms of strongly $\ell$-independent elements $b_{1}, \ldots, b_{r}$ as in (5). By [3, Theorem 1 (3)] we may change the $b_{i}$ 's in such a way that one of them is of the form $\pm f a^{2}$ with $a \in K^{\times}$(notice that any basis of $\left\langle b_{1}, \ldots, b_{r}\right\rangle$ consists of strongly $\ell$-independent elements because the $d$-parameters are all zero).

The algorithm in the proof of Theorem 4.4 (which only requires a finite case distinction for the $r$-tuples $X$ ) yields a 2 -good basis $\gamma_{1}, \ldots, \gamma_{r}$ of a subgroup $H_{X}^{\prime}$ of $H_{X}$ of odd index having same 2-divisibility parameters as $H_{X}$ over $K$ (and over $K\left(\zeta_{4}\right)$ ) by Theorem 3.2. Notice that also $H_{X}^{\prime}$ contains an element of the form $\pm\left(f a^{2}\right)^{2^{d}}$. We may permute the indices $i, j$ as in the proof of Theorem 4.4 and for every $n \in\{1, \ldots, r\}$ we write

$$
\gamma_{n}=(-1)^{F_{n}} \cdot B_{n}^{2^{d_{n}}}, \quad \text { where } \quad B_{n}:=\prod_{i \geq n} b_{i}^{y_{n i}}
$$

for some integers $y_{n i}$ and $F_{n}$, with $y_{n n}$ odd, and where $d_{n}$ is the $d$-divisibility parameter of $\gamma_{n}$ (in particular, $B_{n}$ is strongly 2 -indivisible). To apply Remark 8 


\section{ANTONELLA PERUCCA—PIETRO SGOBBA-SEBASTIANO TRONTO}

we can take as $j$ any index such that $B_{j}$ is of the form $\pm f a^{2}$. If there is no such index, let $k$ be such that $b_{k}$ is of the form $\pm f a^{2}$. Observe that (without using that $b_{k}$ is of the special form) we can write

$$
b_{k} c^{2}=\prod_{i \in J} B_{i} \quad \text { with } \quad c \in K^{\times}
$$

and

$$
k \in J \subseteq\{k, \ldots, r\} .
$$

Let $j:=\max J$ and consider the base change of $H_{X}^{\prime}$ which replaces $\gamma_{j}$ by

$$
\gamma_{j}^{\prime}:=\prod_{i \in J} \gamma_{i}^{2^{d_{j}-d_{i}}}= \pm \prod_{i \in J} B_{i}^{2^{d_{j}}}= \pm\left(f(a c)^{2}\right)^{2^{d_{j}}},
$$

see [3, Proof of Proposition 6]. The $d$-parameter of $\gamma_{j}^{\prime}$ is $d_{j}$, so again we have a 2 -good basis. The $d$-parameter of $\gamma_{j}^{\prime}$ changes over $K\left(\zeta_{4}\right)$ so $j$ is the index as in Remark 8. There we have to use the $h$-parameter of $\gamma_{j}^{\prime}$, which is 0 unless $\sum F_{i}$ is odd, where the sum is over the indices $i \in J$ such that $d_{i}=d_{j}$.

\section{Three divisibility parameters}

\subsection{The case of rank 3}

Let $K$ be a number field, and let $\ell$ be a prime number. Let $b_{1}, b_{2}, b_{3}$ be strongly $\ell$-independent elements in $K^{\times}$, fix some $\zeta \in \mu_{K}$ of order $\ell^{z}$ and let

$$
g_{i}=\zeta^{f_{i}} \prod_{j=1}^{3} b_{j}^{e_{i j}}
$$

where for $i, j \in\{1,2,3\}$ we have integers $e_{i j}$ such that the matrix $\left(e_{i j}\right)$ has rank 3 , and integers $f_{i}$ such that $1 \leq f_{i} \leq \ell^{z}$. Thus $G=\left\langle g_{1}, g_{2}, g_{3}\right\rangle$ is torsion-free and of rank 3 .

We determine the $\ell$-divisibility parameters for all groups of the form

$$
H_{X}:=\left\langle g_{1}^{\ell^{x_{1}}}, g_{2}^{\ell^{x_{2}}}, g_{3}^{\ell^{x_{3}}}\right\rangle,
$$

where $x_{1}, x_{2}, x_{3}$ are non-negative integers. Consider the matrix of exponents associated to the given basis of $H_{X}$ :

$$
\left(\begin{array}{ccc|c}
e_{11} \ell^{x_{1}} & e_{12} \ell^{x_{1}} & e_{13} \ell^{x_{1}} & f_{1} \ell^{x_{1}} \\
e_{21} \ell^{x_{2}} & e_{22} \ell^{x_{2}} & e_{23} \ell^{x_{2}} & f_{2} \ell^{x_{2}} \\
e_{31} \ell^{x_{3}} & e_{32} \ell^{x_{3}} & e_{33} \ell^{x_{3}} & f_{3} \ell^{x_{3}}
\end{array}\right) .
$$


Up to reordering the $b_{j}$ 's and the $g_{i}$ 's (so up to reordering the rows and the columns of the matrix $\left.\left(e_{i j} \ell^{x_{i}}\right)\right)$ we may suppose that

$x_{1}+v_{\ell}\left(e_{11}\right)$ is the minimum of

$$
\left.x_{i}+v_{\ell}\left(e_{i j}\right) \text { for } i, j \in\{1,2,3\} \text { (restricting to } e_{i j} \neq 0\right) .
$$

Similarly, denoting by $\Delta_{i j}$ the $(i, j)$ th minor of the matrix $\left(e_{i j}\right)$, we may suppose that

$x_{2}+v_{\ell}\left(\Delta_{33}\right)$ is the minimum of

$$
x_{2}+v_{\ell}\left(\Delta_{33}\right), \quad x_{2}+v_{\ell}\left(\Delta_{32}\right), \quad x_{3}+v_{\ell}\left(\Delta_{23}\right), \quad x_{3}+v_{\ell}\left(\Delta_{22}\right)
$$

(restricting to $\Delta_{i j} \neq 0$ ).

The first two steps of the algorithm of the proof of Theorem 4.4 give the matrix

$$
\left(\begin{array}{ccc|c}
e_{11} \ell^{x_{1}} & e_{12} \ell^{x_{1}} & e_{13} \ell^{x_{1}} & f_{1} \ell^{x_{1}} \\
0 & \Delta_{33} A_{1} \ell^{x_{2}} & \Delta_{32} A_{1} \ell^{x_{2}} & D_{2} A_{1} \ell^{x_{2}} \\
0 & 0 & \left(\Delta_{22} \Delta_{33}-\Delta_{32} \Delta_{23}\right) A_{2} \ell^{x_{3}} & \left(\Delta_{33} D_{3}-\Delta_{23} D_{2}\right) A_{2} \ell^{x_{3}}
\end{array}\right),
$$

where

$$
D_{i}:=e_{11} f_{i}-e_{21} f_{1}
$$

are minors of the augmented matrix $\left(e_{i j} \mid f_{i}\right)$, and where

$$
A_{1}:=\ell^{-v_{\ell}\left(e_{11}\right)} \quad \text { and } \quad A_{2}:=\ell^{-v_{\ell}\left(e_{11} \Delta_{33}\right)} .
$$

Thus the $d$-parameters for $H_{X}$ are

$$
\begin{aligned}
& d_{1}=x_{1}+v_{\ell}\left(e_{11}\right) \\
& d_{2}=x_{2}+v_{\ell}\left(\frac{\Delta_{33}}{e_{11}}\right) \\
& d_{3}=x_{3}+v_{\ell}\left(\frac{\Delta_{22} \Delta_{33}-\Delta_{32} \Delta_{23}}{e_{11} \Delta_{33}}\right)
\end{aligned}
$$

in non-decreasing order, and corresponding $h$-parameters are

$$
\begin{aligned}
& h_{1}=\max \left(0, z-x_{1}-v_{\ell}\left(f_{1}\right)\right), \\
& h_{2}=\max \left(0, z-x_{2}-v_{\ell}\left(\frac{D_{2}}{e_{11}}\right)\right), \\
& h_{3}=\max \left(0, z-x_{3}-v_{\ell}\left(\frac{\Delta_{33} D_{3}-\Delta_{23} D_{2}}{e_{11} \Delta_{33}}\right)\right) .
\end{aligned}
$$




\section{ANTONELLA PERUCCA—PIETRO SGOBBA—SEBASTIANO TRONTO}

\subsection{Three divisibility parameters}

As observed in Theorem 4.4, the functions $\delta_{k}$ and $\epsilon_{k}$ do not depend on the rank. This means that for a (similarly defined) group of rank $r \geq 1$ the smallest $d$-parameter and corresponding $h$-parameters are $d_{1}, h_{1}$ as above, provided that 1.

$x_{1}+v_{\ell}\left(e_{11}\right)$ is the minimum of $x_{i}+v_{\ell}\left(e_{i j}\right)$ for $i, j \in\{1, \ldots, r\}$

(restricting to the indices for which $e_{i j} \neq 0$ ).

For rank $r \geq 2$ the second smallest $d$-parameter and corresponding $h$-parameters are $d_{2}, h_{2}$ as above, provided that 1 . holds and that

2 .

$$
x_{1}+v_{\ell}\left(\frac{e_{11} e_{22}-e_{21} e_{12}}{e_{11}}\right) \text { is the minimum of } x_{1}+v_{\ell}\left(\frac{e_{11} e_{i j}-e_{i 1} e_{1 j}}{e_{11}}\right)
$$

for $i, j \in\{2, \ldots, r\}$ (restricting to the indices for which $e_{11} e_{i j}-e_{i 1} e_{1 j} \neq 0$ ).

Finally, for rank $r \geq 3$ the third smallest $d$-parameter and a corresponding $h$ parameter are $d_{3}, h_{3}$ as above, provided that 1 . and 2 . hold and that, with the above notation,

3.

$$
\begin{aligned}
& x_{3}+v_{\ell}\left(\frac{\Delta_{22} \Delta_{33}-\Delta_{32} \Delta_{23}}{e_{11} \Delta_{33}}\right) \text { is the minimum of } \\
& x_{i}+v_{\ell}\left(\frac{\left(e_{11} e_{i j}-e_{i 1} e_{1 j}\right)\left(e_{11} e_{22}-e_{21} e_{12}\right)-\left(e_{11} e_{2 j}-e_{21} e_{1 j}\right)\left(e_{11} e_{i 2}-e_{i 1} e_{12}\right)}{e_{11}\left(e_{11} e_{22}-e_{21} e_{12}\right)}\right)
\end{aligned}
$$

for $i, j \in\{3, \ldots, r\}$ (restricting to the indices for which the integer inside the $\ell$-adic valuation is non-zero).

The above conditions 1., 2., 3. hold up to permuting the rows and the columns of the original matrix, i.e., up to permuting the $g_{i}$ 's and the $b_{j}$ 's.

\subsection{Example}

Let $K=\mathbb{Q}\left(\zeta_{3}\right)$ and $\ell=3$. Since the rational primes 2 and 5 are inert in $K$, they are strongly 3 -independent in $K^{\times}$. Consider the group

$$
G=\left\langle\zeta_{3} 2^{3} 5^{9}, 2^{27} 5\right\rangle .
$$

In order to minimize $v_{3}\left(e_{11}\right)$ we let

$$
b_{1}=5, \quad b_{2}=2, \quad g_{1}=2^{27} 5, \quad g_{2}=\zeta_{3} 2^{3} 5^{9}
$$

so that the augmented matrix of exponents becomes

$$
\left(\begin{array}{ll|l}
e_{11} & e_{12} & f_{1} \\
e_{21} & e_{22} & f_{2}
\end{array}\right)=\left(\begin{array}{cc|c}
1 & 3^{3} & 3 \\
3^{2} & 3 & 1
\end{array}\right) .
$$


The $d$-parameters for $G$ are

$$
\begin{aligned}
& d_{1}:=v_{3}\left(e_{11}\right)=0 \\
& d_{2}:=v_{3}\left(\frac{e_{22} e_{11}-e_{12} e_{21}}{e_{11}}\right)=1
\end{aligned}
$$

and corresponding $h$-parameters are

$$
\begin{aligned}
& h_{1}:=\max \left(0,1-v_{\ell}\left(f_{1}\right)\right)=0, \\
& h_{2}:=\max \left(0,1-v_{\ell}\left(\frac{e_{11} f_{2}-e_{21} f_{1}}{e_{11}}\right)\right)=1 .
\end{aligned}
$$

Consider now, for all non-negative integers $x_{1}, x_{2}$, the subgroup

$$
H_{X}:=\left\langle\left(2^{27} 5\right)^{3^{x_{1}}},\left(2^{3} 5^{9}\right)^{3^{x_{2}}}\right\rangle \quad \text { of } G \text {. }
$$

Its augmented matrix of exponents is

$$
\left(\begin{array}{cc|c}
3^{x_{1}} & 3^{x_{1}+3} & 3^{x_{1}+1} \\
3^{x_{2}+2} & 3^{x_{2}+1} & 3^{x_{2}}
\end{array}\right) .
$$

If $x_{1} \leq x_{2}+1$, then we proceed as above and we get

and

$$
d_{1}=x_{1}, \quad d_{2}=x_{2}+1
$$

$$
h_{1}=0, \quad h_{2}=\max \left(0,1-x_{2}\right) .
$$

If $x_{1}>x_{2}+1$, then we let instead

$$
b_{1}=2, \quad b_{2}=5, \quad g_{1}=\zeta_{3} 2^{3} 5^{9}, \quad g_{2}=2^{27} 5,
$$

so that the matrix becomes

and we have

$$
\left(\begin{array}{cc|c}
3^{x_{2}+1} & 3^{x_{2}+2} & 3^{x_{2}} \\
3^{x_{1}+3} & 3^{x_{1}} & 3^{x_{1}+1}
\end{array}\right)
$$

$$
d_{1}=x_{2}+1, \quad d_{2}=x_{1}, \quad \text { and } \quad h_{1}=\max \left(0,1-x_{2}\right), \quad h_{2}=0 .
$$




\section{ANTONELLA PERUCCA—PIETRO SGOBBA-SEBASTIANO TRONTO}

\section{REFERENCES}

[1] DEBRY, C.-PERUCCA, A.: Reductions of algebraic integers, J. Number Theory $\mathbf{1 6 7}$ (2016), 259-283.

[2] PERUCCA, A.: The order of the reductions of an algebraic integer, J. Number Theory, 148 (2015), 121-136.

[3] PERUCCA, A.-SGOBBA, P.-TRONTO, S.: Addendum to: Reductions of algebraic integers [J. Number Theory 167 (2016), 259-283], J. Number Theory 209 (2020), 391-395.

[4] PERUCCA, A.-SGOBBA, P.-TRONTO, S.: The degree of Kummer extensions of number fields, Int. J. Number Theory 17 (2021), no. 5, 1091-1110.

DOI: https://doi.org/10.1142/S1793042121500263

Received October 13, 2020

Accepted August 30, 2021

\section{Antonella Perucca \\ Pietro Sgobba \\ Sebastiano Tronto}

Department of Mathematics

Faculty of Science,

Technology and Medicine

University of Luxembourg

Avenue de la Fonte 6

L-4364 Esch-sur-Alzette

LUXEMBOURG

E-mail: antonella.perucca@uni.lu pietro.sgobba@uni.lu sebastiano.tronto@uni.lu 